\title{
Gestão de custos na visão de Business Process Management: uma simulação com o método de custeio Activity Based Costing
}

\begin{abstract}
Marino Luiz Eyerkaufer Doutorado em Engenharia de Produção pela Universidade Federal de Santa Catarina UFSC Professor da Universidade do Estado de Santa Catarina - UDESC Rua Dr. Getúlio Vargas, 2822. Bela Vista. Ibirama/SC. CEP: 89140-000 E-mail:marino.luiz@udesc.br

Rodrigo Rengel Doutorado em andamento em Contabilidade pela Universidade Federal de Santa Catarina - UFSC Rua Eng. Agronômico Andrei Cristian Ferreira, s/n. Trindade. Florianópolis/SC. CEP: 88040-900 E-mail: rengel.rodrigo@hotmail.com

Rodrigo Wamser Graduação em Ciências Contábeis pela Universidade do Estado de Santa Catarina UDESC

Contador Rua Angelo Richter, s/n. Vila Nova. Lontras/SC. CEP: 89182-000 E-mail: rodrigo_dlg2@hotmail.com
\end{abstract}

\section{RESUMO}

Este estudo objetiva definir a modelagem na visão de Business Process Management (BPM) de um processo de trabalho em uma empresa prestadora de serviços contábeis e seu custeamento por meio do método Activity Based Costing (ABC). A pesquisa é descritiva quanto aos objetivos e qualitativa quanto à abordagem do problema, empírica na forma de um estudo de caso realizado em uma empresa prestadora de serviços contábeis de Santa Catarina. Os resultados demonstram que o método de custeio ABC pode ser aplicado por meio da visão de BPM na mensuração dos processos de trabalho, sendo útil tanto para medição dos valores dos processos, quanto como instrumento para o gerenciamento das diversas atividades das empresas prestadoras de serviços contábeis. Conclui-se que o principal desafio da gestão de custos na visão do BPM em processos de trabalho é a implantação do modelo, bem como a sua onerosidade nesta fase, por outro lado, as organizações que persistem com este modelo de gestão unificado ao método de custeio $A B C$, reúnem informações relevantes e precisas que permitem redução de custos, assim como a melhoria contínua dos processos e consequentemente a prestação dos serviços de qualidade. 
Gestão de custos na visão de Business Process Management: uma simulação com o método de custeio Activity Based Costing

Marino Luiz Eyerkaufer, Rodrigo Rengel, Rodrigo Wamser

Palavras-chave: Gestão de custos. Business Process Management. Activity Based Costing.

\title{
Cost management in Business Process Management vision: a simulation with the Activity Based Costing method
}

\begin{abstract}
This study aims to define the model used in the Business Process Management (BPM) vision of a work process in a company that provides accounting services and its cost through the Activity Based Costing (ABC) method. The research is descriptive in terms of objectives and qualitative in approach, bein an empirical study in the form of a case study carried out in a company that provides accounting services in Santa Catarina. The results show that $A B C$ costing method can be applied through the BPM vision in the measurement of work processes, being useful both for the measurement of process values and as an instrument for the management of the various activities of companies providing accounting services. We concluded that the main challenge of cost management in the BPM vision in work processes is the implantation of the model as well as its onerosity in this phase, on the other hand, the organizations that persist with this management model unified to the costing method $A B C$, gather relevant and accurate information that allow cost reduction, as well as continuous improvement of processes and consequently the provision of services.
\end{abstract}

Keywords: Costs Management. Business Process Management. Activity Based Costing.

\section{Gestión de costes en la visión de Business Process Management: una simulación con el método Activity Based Costing}

\section{RESUMEN}

Este estudio tiene como objetivo definir el modelado en la visión de Business Process Management (BPM) de un proceso de trabajo en una empresa que brinda servicios contables y su costeo por medio del método Activity Based Costing (ABC). La investigación es descriptiva en cuanto a objetivos y cualitativa en cuanto al abordaje del problema, empírica en forma de estudio de caso realizado en una empresa que presta servicios contables en Santa Catarina. Los resultados demuestran que el método de costeo $A B C$ se puede aplicar por medio de la visión BPM en la medición de procesos de trabajo, siendo útil tanto para medir valores de proceso como como instrumento para la gestión de las diversas actividades de los proveedores de servicios contables. Se concluye que el principal desafío de la gestión de costos a la vista de BPM en los procesos de trabajo es la implementación del modelo, así como su costo en esta fase, 
Gestão de custos na visão de Business Process Management: uma simulação com o método de custeio Activity Based Costing

Marino Luiz Eyerkaufer, Rodrigo Rengel, Rodrigo Wamser

por otro lado, organizaciones que persisten con este modelo de gestión unificado con el método de costeo $A B C$, recopila información relevante y veraz que permite la reducción de costos, así como la mejora continua de los procesos y en consecuencia la prestación de servicios de calidad.

Palabras clave: Gestión de costs. Business Process Management. Activity Based Costing.

\section{INTRODUÇÃO}

As organizações estão atualmente inseridas em um ambiente altamente competitivo, por conta da rápida evolução tecnológica que vem ocorrendo desde o final do século XX. A fim de sobreviverem e manterem competitivas as empresas que buscam reformular continuamente suas estratégias e se preocupam cada vez mais com sua gestão de custos (Megliorini, 2012).

Porter (1989) aponta a liderança em custos como um dos pilares de sua Teoria das Forças Competitivas. Machado e Souza (2006) entendem que uma gestão de custos eficaz, necessariamente engloba atividades de planejamento, execução e controle eficientes. Ainda de acordo com Machado e Souza (2006), busca-se de variadas maneiras a redução de custos e aumento da produtividade das organizações, tais como os métodos de custeio, os quais são utilizados a fim de reduzir custos, obter informações, suprimir desperdícios e aprimorar os processos (Abbas, Gonçalves \& Leoncine, 2012).

Um dos modelos de gestão de custos é o método Activity Based Costing (ABC), também conhecido como custeio baseado em atividade, que surgiu no final dos anos 80, idealizado por Coopers e Kaplan. Para Cogan (2010), o custeio ABC é diferente dos métodos de custeios tradicionais, pela forma com que são acumulados os custos. $O$ sistema tradicional utiliza-se de um modelo de acumulação de dois estágios. Primeiramente os custos são aglomerados por função ou departamento e depois rateados pelos produtos por meio de um simples fator volumétrico de medição. $O A B C$ 
Gestão de custos na visão de Business Process Management: uma simulação com o método de custeio Activity Based Costing Marino Luiz Eyerkaufer, Rodrigo Rengel, Rodrigo Wamser

tem os recursos e as atividades como foco, enquanto o custeio tradicional tem os produtos como geradores de custos.

Anand (2004) entende o método de custeio ABC como uma fórmula de custeio mais moderna, que objetiva a redução dos problemas da atribuição dos custos indiretos aos produtos. Uma das características predominantes e que diferencia o método $A B C$ dos demais modelos de custeio é o fato de que os custos são consumidos pelas atividades, e que essas consomem os recursos, assim podendo ser diretamente interligado ao Business Process Management (BPM).

Segundo Scucuglia (2011), o BPM altera significativamente o modo como a cadeia de valor é encarada e propõe uma visão interfuncional de como os processos ponta-a-ponta poderiam ser mais bem geridos, visando eliminar os efeitos maléficos dos conflitos internos entre os departamentos. Conforme Lagioia, Ribeiro Filho, Falk, Libonati e Lopes (2008), a implementação de gestão por processos em uma organização faz com que essa tenha maior controle interno, bem como redução dos custos, devido a visão geral da estrutura empresarial, possibilitando cortes em retrabalhos e processos indevidos.

As necessidades de as organizações conhecerem seus processos e os custos deles fazem desses procedimentos de controle, cada vez mais fundamental para permanência de mercado, devido concorrência acirrada e precificação dos produtos sendo definida pela demanda, obriga-se então as organizações a reduzirem custos para alcançarem maior lucratividade. Abbas, Gonçalves e Leoncine (2012) afirmam que o método de custeio $A B C$, permite esse controle, rastreamento e gestão de processos, bem como ferramenta de gestão, visualizando os recursos utilizados para cada atividade.

Diante do exposto, tem-se como problema de pesquisa o seguinte questionamento: Qual é o alinhamento do método de custeio $A B C$ no custeamento de processos de trabalho (BPM) em empresas prestadoras de serviços contábeis? Para responder isso, o objetivo deste estudo é definir a modelagem na visão de Business 
Gestão de custos na visão de Business Process Management: uma simulação com o método de custeio Activity Based Costing Marino Luiz Eyerkaufer, Rodrigo Rengel, Rodrigo Wamser

Process Management (BPM) de um processo de trabalho em uma empresa prestadora de serviços contábeis, e seu custeamento por meio do método $A B C$.

Destarte, justifica-se esta pesquisa por preencher a lacuna observada na literatura, ao analisar processos de trabalho (BPM) por meio de uma simulação a partir do método $A B C$. Desse modo, proporciona-se uma modelagem da estrutura de trabalho que se enquadre com as necessidades e características observadas no caso. Portanto, facilita-se a identificação dos custos da atividade analisada. Justifica-se ainda por contribuir no campo prático ao fornecer informações aos gestores do impacto dos custos por processos, bem como analisar os controles internos das organizações, auxiliando a busca por excelência dos serviços contábeis.

Além dessa seção introdutória, este estudo está dividido em outras sete seções. Em seguida, apresenta-se a revisão da literatura de gestão de custos; seguida da gestão por processos (BPM), na seção quatro apresenta-se o método de custeio $A B C$ na gestão por processos; a seção cinco aborda os procedimentos metodológicos; a apresentação e análise de uma aplicação do ABC em BPM aparecem na sexta seção; as conclusões na sétima e, por fim, as referências bibliográficas.

\section{REFERENCIAL TEÓRICO}

\subsection{Gestão de Custos}

Perante as deficiências dos sitemas de custeio tradicionais, que muitas vezes estão relacionados à apuração distorcida dos custos e comumente utilizado apenas o volume da produção como critério de rateio, faz surgir a necessidade de desenvolver uma gestão de custos mais efetiva e rigorosa. Inicialmente as empresas prendiam-se apenas com a medição dos custos, mas rapidamente surgiu a necessidade de realizar a gestão de custos para a tomada de decisão (Silva, 1999).

Segundo Bruni e Famá (2004) as relações e interações entre a empresa e o ambiente em que essa está inserida, além da realização do processo de construção do 
Gestão de custos na visão de Business Process Management: uma simulação com o método de custeio Activity Based Costing Marino Luiz Eyerkaufer, Rodrigo Rengel, Rodrigo Wamser

futuro alinhada ao processo de gestão empresarial referem-se à estratégia da organização.

A preocupação com os custos dentro das empresas em um contexto estratégico na gestão dos negócios traz vantagens competitivas às organizações. Com isso, a gestão de custos implica na apresentação de uma nova visão da contabilidade gerencial, sendo essa, ligada a estratégias empresariais (Ciotti, 2011). Porter (1980) por sua vez, define estratégia empresarial como a maneira que um negócio vai competir no mercado, alinhando as metas estratégicas e gerenciais para alcançar os objetivos da organização.

Para Perez Junior, Oliveira e Costa (2003), integrar a gestão estratégica aos processos da administração e da controladoria, a partir da visualização da organização por meio de novos e diferentes ângulos, proporciona vantagens que se apresentam como um importante instrumento para tomada de decisão. Ainda, a focalização dos esforços de melhoria com resultados mensuráveis e o aprimoramento na capacidade da empresa criar e agregar valores. Com isso, Ciotti (2011) conclui que a gestão de custos é uma ferramenta fundamental para que as organizações consigam atingir vantagens competitivas.

A gestão de custos simboliza uma inovação perante 0 atendimento da necessidade de informação das organizações como elemento estratégico, em um mercado em constante transformação (Cunha, Borgert \& Ferrari, 2012). Para Diehl, Capalonga, Souza e Zanini (2009), toda organização busca alcançar melhores resultados, para isso, são necessárias estratégias para manipular os recursos internos, aproveitando as oportunidades no ambiente externo, ou seja, realizando a gestão por processos da organização.

\subsection{Gestão por Processos}

O gerenciamento por processos de negócios, oriundo da denominação em inglês Business Process Management (BPM) é uma nova visão das operações de negócio, que vai além das estruturas funcionais tradicionais. Essa visão envolve todo o trabalho 
Gestão de custos na visão de Business Process Management: uma simulação com o método de custeio Activity Based Costing Marino Luiz Eyerkaufer, Rodrigo Rengel, Rodrigo Wamser

executado até a entrega do produto ou serviço, possibilitando o entendimento das operações e atuações de cada participante de todos os processos de negócio (BPM CBOK, 2013).

Para que se compreenda o BPM é preciso entender o que é um processo de negócio. Para Oliveira (2010), um processo é desenvolvido por meio de uma sequência de atividades que resultam no produto da organização, seja um bem ou serviço. Também pode ser visto como a execução de atividades em que se tem uma entrada, uma transformação e uma saída, resultando no alcance de metas.

Os processos de negócio são classificados em três tipos: (i) os processos primários, que são aqueles que formam as atividades essenciais da organização e agregam valor para os clientes; (ii) os processos de suporte, que são aqueles caracterizados por suportar as atividades essenciais a organização, ou seja, são aqueles que possuem um conjunto de atividades ligadas à infraestrutura e/ou ao gerenciamento de recursos que viabilizam o funcionamento dos subsistemas e; (iii) os processos de gerenciamento, que são aqueles responsáveis em medir, monitorar e controlar as atividades do negócio (BPM CBOK, 2013).

Com os processos estabelecidos, a etapa seguinte para se obter vantagens na utilização do BPM é a gestão, que para De Sordi (2005) é desempenhar as atividades de monitoramento e controle dos recursos da organização, de forma que estabeleça evolução dos mesmos, como apresenta a Figura 1. 
Gestão de custos na visão de Business Process Management: uma simulação com o método de custeio Activity Based Costing Marino Luiz Eyerkaufer, Rodrigo Rengel, Rodrigo Wamser

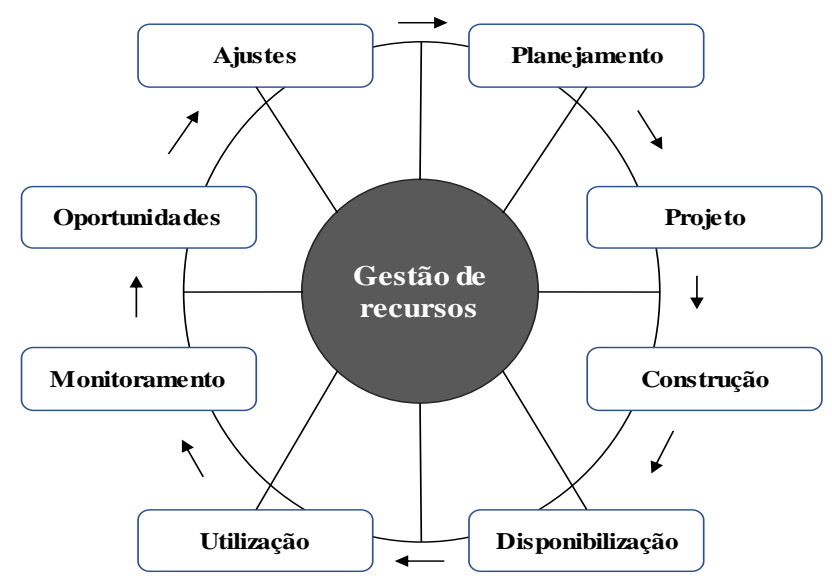

Figura 1. Processo de gestão de recursos

Fonte: De Sordi (2005).

A gestão por processos de trabalho é segundo De Sordi (2005) o meio conciliador dos ativos e interesses da organização, de forma a se obter melhores resultados e desempenhos, como descrito na Figura 2.

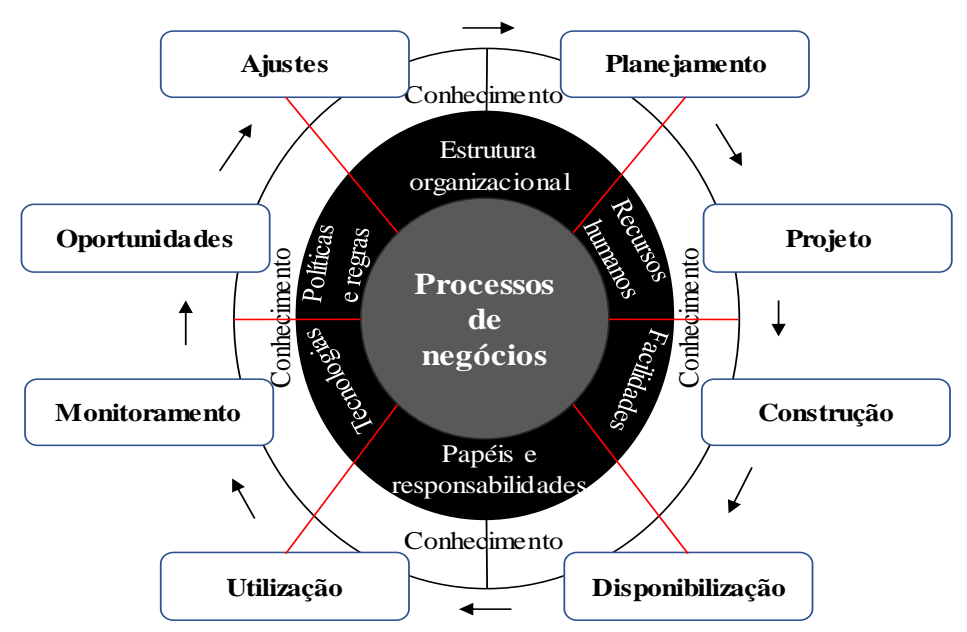

Figura 2. Processos de negócios e seus ativos a serem geridos

Fonte: De Sordi (2005), adaptado de Burlton (2001). 
Gestão de custos na visão de Business Process Management: uma simulação com o método de custeio Activity Based Costing Marino Luiz Eyerkaufer, Rodrigo Rengel, Rodrigo Wamser

O gerenciamento ponta a ponta de processos de negócios e a orquestração das atividades ao longo das funções de negócio, são a essência do BPM sendo o que o diferencia do gerenciamento funcional tradicional (BPM CBOK, 2013).

De acordo com Burlton (2001), a gestão de processos denominada como BPM, busca evidenciar as atividades de: modelar, documentar, simular, automatizar, medir, monitorar e melhorar os processos do negócio visando alcançar os resultados prédeterminados pelos objetivos organizacionais. Pode-se concluir que o BPM é um modelo de gestão, que busca administrar todo o ciclo produtivo da organização, de melhoria contínua, o qual deve estar alinhado perante os objetivos do negócio e, que para tanto, transcorre os diferentes departamentos, áreas e unidades de toda organização.

\subsection{Método de custeio baseado em atividades na gestão por processos}

Os métodos de custeios são considerados fundamentais para o gerencimento estratégico e tomada de decisão, dos quais, os modelos mais utilizados para determinar o custo do produto/serviço são: o custeio baseado em atividades (ABC), custo padrão, custeio por absorção, custeio variável/direto e o custo meta, segundo Carareto, Jayme, Tavares e Vale (2006). Para o setor de prestação de serviços contábeis, do qual seu produto final é intangível, faz com que sua mensuração se torne mais difícil e complexa (Beuren, Coronetti \& Sousa, 2012; Silva, Ramalho \& Jordão, 2015).

Segundo Gasparetto (1999), nos sistemas de custeio tradicionais os custos diretos, correspondentes a mão de obra e materiais, representavam a maior parte dos custos das empresas, havendo pouca representatividade dos custos indiretos nos custos totais. Com a mudança no perfil competitivo das empresas foram criadas atividades de suporte que têm maior importância e consomem mais recursos, como as atividades de marketing, logística, distribuição, qualidade e outras. Apurar os custos dessas atividades inseriu maior complexidade nos sistemas de gerenciamento de custos (Gasparetto, 1999). 
Gestão de custos na visão de Business Process Management: uma simulação com o método de custeio Activity Based Costing Marino Luiz Eyerkaufer, Rodrigo Rengel, Rodrigo Wamser

O ABC surgiu com o objetivo de sanar as distorções provocadas pelos modelos de custeio tradicionais e suprir a precariedade de informações gerenciais proporcionadas por esses modelos (Coelho, 2011; Tabosa, Júnior, Rodrigues, \& Pinheiro, 2008). A importância do efeito dos custos indiretos sobre o lucro e a competitividade das empresas iniciou a discussão que deu origem à metodologia do $A B C$, uma metodologia que reconhece a complexidade e variabilidade dos processos produtivos contemporâneos, de acordo com Gasparetto (1999).

Custos são vistos tradicionalmente como os recursos consumidos por serviços ou produtos. Já o método de custeio $A B C$, possui uma perspectiva diferente, para este, serviços ou produtos consomem atividades e estas por sua vez consomem recursos (Bonacim \& Araujo, 2010). Diante do exposto, para a devida compreensão do ABC, fazse necessário o entendimento do termo atividade. Martins (2003) considera como atividade, a combinação de recursos humanos, materiais, tecnológicos e financeiros para a produção de determinado produto ou serviço.

De acordo com Santos, Schmidt, Pinheiro e Nunes (2006), o custeio ABC é o método que surge para dar o suporte gerencial para as organizações, uma vez que para se adequarem a nova realidade competitiva, as empresas necessitam não só de informações que quantificam seus estoques, mas também informações confiáveis para auxiliar na tomada de descisão em todo âmbito produtivo. A maior disposição de informações gerenciais podem trazer para a empresa vantagens competitivas.

O ABC utiliza uma visão horizontal da empresa (os processos), associada à visão vertical tradicionalmente utilizada (organograma), para fazer a análise dos processos que cruzam a empresa e custear as atividades que os integram. O foco deixa de ser somente a produção, e se estende para toda a cadeia de atividades que se relacionam para que o produto seja fabricado (Gasparetto, 1999).

$O$ Custeio $A B C$ procura monitorar os gastos da organização e suas rotas de consumo dos recursos para chegar ao serviço ou produto (Nakagawa, 2001). Seguindo os pensamentos do autor, esse rastreamento oferecido pelo $A B C$ pode ser dividido em duas etapas: a primeira, identifica, classifica e mensura o consumo dos recursos em 
Gestão de custos na visão de Business Process Management: uma simulação com o método de custeio Activity Based Costing

Marino Luiz Eyerkaufer, Rodrigo Rengel, Rodrigo Wamser

cada atividade; e a segunda etapa, procura identificar como os produtos consomem as atividades.

Sendo assim, as informações obtidas pelo ABC possibilitam o gerenciamento das atividades que envolvem o processo produtivo da organização, sendo assim, proporcionando a gestão por processos. Este mecanismo de custeio do ABC pode ser melhor compreendido com base na Figura 3.

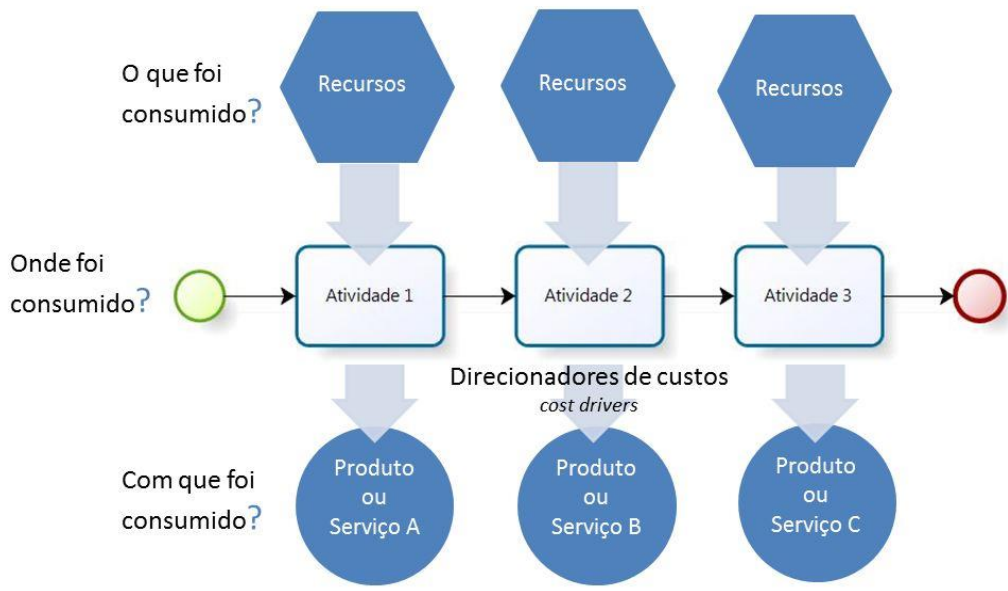

Figura 3. Mecanismo de custeio ABC

Fonte: Luz Planilhas Empresariais (2017).

Machado e Souza (2006), apontam que organizações objetivam a redução de custos e, além disso, preocupam-se com o aumento da produtividade das organizações. O estudo desenvolvido por Lagioia et al. (2008) tratou sobre a gestão por processos e como essa gera melhoria de qualidade e redução de custos, realizado a partir de um caso da unidade de ortopedia e traumatologia do hospital das clínicas da Universidade Federal de Pernambuco. Por meio desse, os autores afirmaram que na utilização do gerenciamento dos processos, percebeu-se diminuições nos custos e melhorias internas. 
Gestão de custos na visão de Business Process Management: uma simulação com o método de custeio Activity Based Costing Marino Luiz Eyerkaufer, Rodrigo Rengel, Rodrigo Wamser

Mesma visão pode ser percebida na pesquisa de Dos Santos e Robaina (2014), que apresenta o impacto do BPM nos custos organizacionais, assim como a importância da gestão por processos para criar processos mais eficientes e eficazes, tornando-se uma prática estratégica e competitiva em prol da organização perante o mercado altamente competitivo, além de comprovar o impacto positivo sobre os resultados financeiros organizacionais.

\section{PROCEDIMENTOS METODOLÓGICOS}

Esta pesquisa pode ser classificada como descritiva perante os objetivos, qualitativa quanto à abordagem do problema, e sobre os procedimentos, consistindo em um estudo de caso desenvolvido em uma empresa prestadora de serviços contábeis de Santa Catarina. Para Yin (2005), o estudo de caso é uma maneira de se realizar pesquisas empíricas que analisam eventos contemporâneos em seus contextos e realidades.

Utilizou-se para a coleta de dados entrevistas não estruturadas, análise documental e observação participante. A pesquisa de campo utilizou-se de uma amostragem não probabilística, realizada de forma intensiva por um pesquisador que efetuou a coleta de dados na empresa escolhida, todos os dias por um período de 4 meses, numa jornada de 4 horas diárias. Assim, foram 83 dias de coleta de dados e de análises in loco dos resultados.

As entrevistas não estruturadas foram realizadas com os seis funcionários da empresa. Essas entrevistas objetivaram compreender os processos desempenhados em cada departamento. Beuren e Raupp (2006) afirmam que esse modelo de entrevista possibilita ao entrevistado autonomia para desenvolver suas respostas, não se limitando a opções e caminhos preestabelecidos, assim explorando mais profundamente os fatos pesquisados.

A análise documental, em contrapartida, objetivou analisar os documentos de cada departamento da empresa, buscando identificar as atividades desenvolvidas e 
Gestão de custos na visão de Business Process Management: uma simulação com o método de custeio Activity Based Costing Marino Luiz Eyerkaufer, Rodrigo Rengel, Rodrigo Wamser

conhecer melhor o ciclo operacional, bem como os custos desses procedimentos. Este procedimento de análise baseia-se em materiais que não receberam nenhum tipo de interferência por parte de estudiosos e por isso podem ser estruturados com base no objetivo que a pesquisa busca alcançar. De acordo com Raupp e Beuren (2011), o objetivo é selecionar, tratar e interpretar a informação em seu estado bruto, buscando extrair dela o sentido para o estudo, isto é, agregando valor.

Em relação à observação participante, os pesquisadores possuíram total independência para acompanhar e observar a execução das atividades dos funcionários de cada departamento. Dessa forma surgiram algumas trocas de informações importantes, o que permitiu a obtenção de conhecimento relevante sobre os processos internos da Empresa. Em alguns casos, como especialmente no Departamento Administrativo/Financeiro, os pesquisadores tiveram a possibilidade de desempenhar a atividade de controle e gerenciamento dos custos e processos internos da empresa. Essa autonomia para executar as teorias estudadas no ambiente real proporcionou uma melhor avaliação dos processos, tempos, custos e demais variáveis dos serviços prestados.

Desenvolveu-se, assim, uma triangulação dos dados coletados por meio das técnicas de coleta de dados descritas. A triangulação dos dados é o processo de confrontação dos dados coletados utilizando-se de diferentes técnicas de coleta, validando a confiabilidade da pesquisa (Yin, 2005).

Para o desenvolvimento da modelagem do processo de trabalho na visão do BPM, utilizou-se o software Bizagi Modeler. Para tratamento e interpretação dos dados, tabulou-se esses em planilhas eletrônicas para identificação dos custos por meio do método de custeio $A B C$. O processo de trabalho analisado por este estudo consiste no arquivamento dos documentos contábeis.

A escolha por uma empresa prestadora de serviços contábeis deve-se ao fato que esse tipo de organização possui departamentalização dos serviços prestados, os quais, por fim são unificados no serviço final. Justifica-se a escolha desta empresa dentre tantas do segmento, devido a abertura à esta pesquisa. Outro fator determinante 
Gestão de custos na visão de Business Process Management: uma simulação com o método de custeio Activity Based Costing Marino Luiz Eyerkaufer, Rodrigo Rengel, Rodrigo Wamser

para a escolha deste modelo de organização deve-se à necessidade de processos alinhados, por atuação regulamentada por Órgãos além de prazos predefinidos para apuração de impostos e demais atividades. Dentre os processos, a escolha pelo processo de arquivo, justifica-se devido ao englobamento de todos os setores do escritório, atividades de descarte dos documentos, bem como processos de controle interno.

\section{SIMULAÇÃO DO ACTIVITY BASED COSTING EM BUSINESS PROCESS MANAGEMENT}

Apresenta-se nesta seção o estudo de caso abordado na pesquisa empírica. Trata-se de uma empresa que atua como prestadora de serviços contábeis na região do Alto Vale do Itajaí, em Santa Catarina. No intuito de resguardar informações sigilosas sobre a empresa estudada, utiliza-se o pseudônimo "Empresa Alfa". Conforme a Lei Complementar № 123/06, essa empresa pode ser considerada como de pequeno porte, por auferir receita anual acima de $R \$ 360$ mil e abaixo de $R \$ 3,6$ milhões (Brasil, 2006).

A Empresa Alfa foi fundada na década de 70 e atua no setor de serviços contábeis, como dito anteriormente. Basicamente, pode ser segregada em seis setores principais: Fiscal, Recursos Humanos, Contábil, Legalizações, Atendimento e Administrativo/Financeiro. Mesmo sendo considerada uma empresa de pequeno porte, a Empresa Alfa possui centenas de clientes espalhados pela região do Alto Vale do Itajaí em Santa Catarina. Para o desenvolvimento desta pesquisa, os pesquisadores coletaram as informações necessárias referentes aos processos realizados em cada departamento. Esses custos foram identificados in loco, por meio da observação participante e de entrevistas não estruturadas. Os dados e informações referentes aos custos foram coletados a partir de relatórios de controles internos da empresa em seu ERP. 
Gestão de custos na visão de Business Process Management: uma simulação com o método de custeio Activity Based Costing Marino Luiz Eyerkaufer, Rodrigo Rengel, Rodrigo Wamser

De acordo com Pizza (2012), a metodologia do BPM é comprovadamente um investimento seguro e de rápido retorno, pois auxilia as empresas na identificação da relevância estratégica de seus processos e na execução de decisões para obtenção de vantagens competitivas. Além disso, Dos Santos e Robaina (2014) afirmam que havendo uma melhor harmonização dos processos internos, assim como melhor utilização dos recursos organizacionais, pode-se ter uma redução nos custos da empresa.

Optou-se, portanto, pelo detalhamento horizontal, tendo-se em vista a metodologia de controle e análise abordada pelo BPM, do qual foi compreendido na sua maioria por duas tarefas ou mais. O processo foi modelado para cada departamento por meio do Bizagi Modeler, no qual detalhou-se todas as tarefas pertinentes a cada processo. O processo de arquivamento pode ser visualizado pela Figura 4.

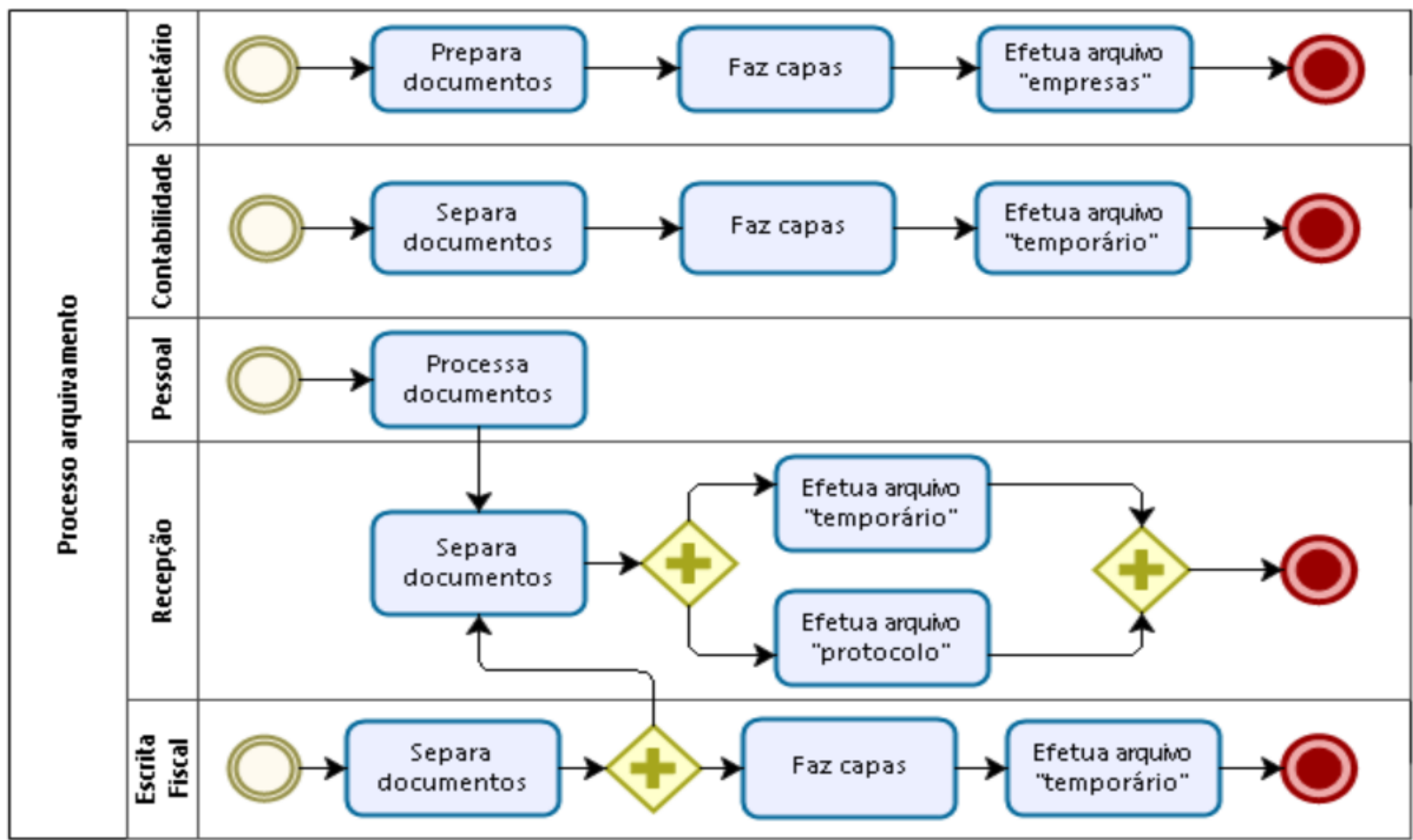

Figura 4. Processos de arquivamento

Fonte: Elaborada pelos autores. 
Gestão de custos na visão de Business Process Management: uma simulação com o método de custeio Activity Based Costing Marino Luiz Eyerkaufer, Rodrigo Rengel, Rodrigo Wamser

Uma vez evidenciado o processo de arquivamento, faz-se necessário a identificação e mensuração dos custos de cada uma das atividades. Para Costa (2013), uma das funções mais importantes de um sistema de gerenciamento de custos por processos, é acumular custos de produtos e serviços incluindo os custos de sua atividade e os organizar em informações relevantes, principalmente nos processos de elaboração, a fim de auxiliar no processo decisório.

Para a evidenciação dos custos do processo Arquivamento, os pesquisadores levantaram as informações de custos referentes aos recursos existentes e utilizados em cada departamento da empresa, mensuráveis a partir do reconhecimento dos seus respectivos processos de execução. A Tabela 1 apresenta os itens, classificações, valores e direcionadores dos custos presentes no processo de trabalho analisado.

Tabela 1

Itens de custo, classificação e seus direcionadores

\begin{tabular}{lccc}
\hline Recursos /atividades & Valores (R\$) & Classificação & Direcionador \\
\hline Salários e Encargos & $\mathrm{R} \$ 12.107,22$ & Indireto & Tempo médio (minutos) \\
Depreciação Imóvel & $\mathrm{R} \$ 1.000,00$ & Indireto & Metro quadrado \\
Depreciação Móveis & $\mathrm{R} \$ 125,00$ & Direto & Apropriação Direta \\
IPTU & $\mathrm{R} \$ 728,00$ & Indireto & Metro quadrado \\
Energia Elétrica & $\mathrm{R} \$ 350,00$ & Indireto & Quilowatt-hora \\
Material de Expediente & $\mathrm{R} \$ 90,38$ & Direto & Apropriação Direta \\
Material de Limpeza & $\mathrm{R} \$ 15,00$ & Indireto & Metro quadrado \\
Serviços de Terceiros & $\mathrm{R} \$ 1.120,00$ & Indireto & Metro quadrado \\
\hline
\end{tabular}

Nota. Fonte: Dados da pesquisa (2019).

Com base nas informações obtidas durante o reconhecimento dos custos e detalhamento das tarefas do processo de arquivamento, sendo executado por cada departamento, faz se necessário identificar os direcionadores de custos. Os custos com a depreciação de móveis foram alocados de forma direta. Os custos com IPTU, 
Gestão de custos na visão de Business Process Management: uma simulação com o método de custeio Activity Based Costing Marino Luiz Eyerkaufer, Rodrigo Rengel, Rodrigo Wamser

depreciação do imóvel, serviços de terceiros e limpeza, foram alocados com base nos metros quadrados ocupados pelo arquivo físico.

Os custos incorridos com a mão de obra, por sua vez, foram apropriados de acordo com o tempo consumido durante todo o processo. Os custos com materiais de escritório foram alocados a partir da padronização de consumo adotado pela empresa e a energia elétrica pelo consumo em $\mathrm{KW} / \mathrm{H}$ dos equipamentos eletrônicos da área corresponde ao arquivo físico. Já os custos que não puderam ser identificados de forma precisa a uma determinada tarefa do processo de arquivamento, então foram excluídos do cálculo a fim de se eliminar possíveis vieses.

Para o cálculo do custo da mão de obra foram realizadas observações intensivas e medições de tempo consumidas para a execução de todas as tarefas necessárias demostradas no mapeamento do processo. Tendo-se, assim, o custo base da mão de obra do processo por departamento. Para o custo total, cada processo foi multiplicado por cento e cinquenta, número de vezes que as tarefas são executadas por cada departamento para arquivar todos os clientes mensalmente, como apresenta-se na Tabela 2.

Tabela 2

Custo médio da Mão de Obra direta das tarefas por minuto

\begin{tabular}{|c|c|c|c|c|c|c|c|c|c|c|}
\hline 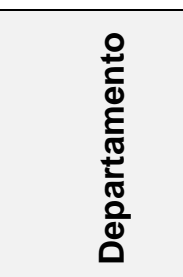 & $\begin{array}{l}\frac{\pi}{0} \\
\frac{0}{0} \\
0 \\
\frac{0}{9} \\
0\end{array}$ & 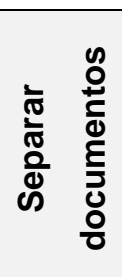 & $\begin{array}{l}\frac{\pi}{0} \\
\frac{\pi}{0} \\
\frac{\pi}{0} \\
\frac{\pi}{0} \\
\frac{0}{0} \\
0 \\
0\end{array}$ & 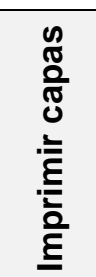 & $\begin{array}{l}\frac{\pi}{0} \\
\frac{10}{\pi} \\
\frac{\pi}{0} \\
\frac{0}{0} \\
03\end{array}$ & 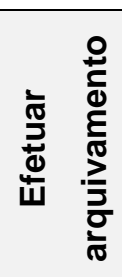 & 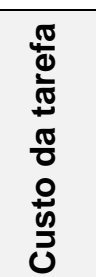 & 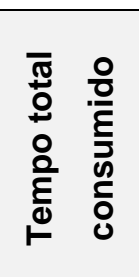 & 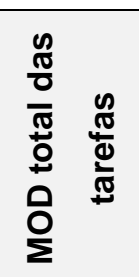 & 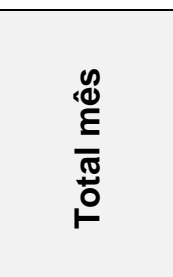 \\
\hline Fiscal & $\mathrm{R} \$ 0,42$ & 0,67 & 0,27 & 0,92 & 0,38 & 1,08 & 0,45 & 2,67 & $\mathrm{R} \$ 1,11$ & $\mathrm{R} \$ 166,06$ \\
\hline Contábil & $R \$ 0,38$ & 1,33 & 0,50 & 1,30 & 0,49 & 1,58 & 0,60 & 4,22 & $\mathrm{R} \$ 1,59$ & $\mathrm{R} \$ 238,14$ \\
\hline Recepção & $\mathrm{R} \$ 0,13$ & & 0,63 & & & 2,50 & 0,31 & 7,50 & $\mathrm{R} \$ 0,94$ & $R \$ 141,38$ \\
\hline
\end{tabular}

Nota. Fonte: Dados da pesquisa (2019). 
Gestão de custos na visão de Business Process Management: uma simulação com o método de custeio Activity Based Costing Marino Luiz Eyerkaufer, Rodrigo Rengel, Rodrigo Wamser

Percebe-se que não foram considerados os custos de mão de obra do departamento pessoal devido a não participação dele na tarefa de arquivamento. $A$ recepção é responsável pelo arquivamento dos documentos da folha de pagamento. Por fim, na Tabela 3, apresenta-se o custo total mensal para o arquivamento de clientes na Empresa Alfa, obtido a partir da unificação de todos os recursos necessários continuamente e consumidos durante o processo.

Tabela 3

Custo total do Arquivamento

\begin{tabular}{lcccc}
\hline Recursos /atividades & Valores (R\$) & $\begin{array}{c}\text { Custo unitário } \\
\text { das atividades } \\
\mathbf{( R \$ )}\end{array}$ & $\begin{array}{c}\text { Quantidade } \\
\text { Consumida }\end{array}$ & $\begin{array}{c}\text { Custo Total por } \\
\text { atividade (R\$) }\end{array}$ \\
\hline Departamento Fiscal & $\mathrm{R} \$ 5.480,11$ & $\mathrm{R} \$ 1,11$ & 150 & $\mathrm{R} \$ 166,50$ \\
Departamento Contábil & $\mathrm{R} \$ 4.970,33$ & $\mathrm{R} \$ 1,59$ & 150 & $\mathrm{R} \$ 238,50$ \\
Recepção & $\mathrm{R} \$ 1.656,78$ & $\mathrm{R} \$ 0,94$ & 150 & $\mathrm{R} \$ 141,00$ \\
Depreciação Imóvel & $\mathrm{R} \$ 1.000,00$ & $\mathrm{R} \$ 7,69$ & $34,61 \%$ & $\mathrm{R} \$ 346,10$ \\
Depreciação Móveis & $\mathrm{R} \$ 125,00$ & $\mathrm{R} \$ 2,78$ & $100 \%$ & $\mathrm{R} \$ 125,00$ \\
IPTU & $\mathrm{R} \$ 728,00$ & $\mathrm{R} \$ 5,60$ & $34,61 \%$ & $\mathrm{R} \$ 251,96$ \\
Energia Elétrica & $\mathrm{R} \$ 350,00$ & $\mathrm{R} \$ 0,58$ & 38,88 & $\mathrm{R} \$ 22,72$ \\
Material de Expediente & $\mathrm{R} \$ 90,38$ & $\mathrm{R} \$ 0,60$ & $100 \%$ & $\mathrm{R} \$ 90,38$ \\
Material de Limpeza & $\mathrm{R} \$ 15,00$ & $\mathrm{R} \$ 0,12$ & $34,61 \%$ & $\mathrm{R} \$ 5,19$ \\
Serviços de Terceiros & $\mathrm{R} \$ 1.120,00$ & $\mathrm{R} \$ 8,62$ & $34,61 \%$ & $\mathrm{R} \$ 387,63$ \\
\hline
\end{tabular}

Nota. Fonte: Dados da pesquisa (2019).

Com base nos resultados empíricos, constatou-se que a organização pesquisada apresenta um custo total da tarefa arquivamento de $\mathrm{R} \$ 1.774,98$ por mês. Porém, desse valor, 26,54\% se referem às taxas de depreciação, que não são desembolsáveis pela organização, sendo assim, pode-se considerar que os dispêndios financeiros mensais são de $R \$ 1.303,88$. Destacam-se ainda, os custos de mão de obra, os quais apesar de demandarem pouco tempo para efetuar as tarefas necessárias do arquivamento, são os maiores custos do processo, totalizando $R \$ 546,00$ por mês, equivalente a $30,76 \%$ do custo 
Gestão de custos na visão de Business Process Management: uma simulação com o método de custeio Activity Based Costing

Marino Luiz Eyerkaufer, Rodrigo Rengel, Rodrigo Wamser

total do processo. Os demais custos totalizam $\mathrm{R} \$ 757,88$ por mês, o que representa $42,70 \%$ do total.

Além das perspectivas financeiras, com base na observação participante realizada por este estudo, percebe-se que a partir da modelagem e desenho do BPM, melhorou-se a qualidade do processo de trabalho arquivamento, haja vista que esse proporcionou uma estrutura para sua realização, reduzindo as possibilidades de desvios e perdas de documentos.

Por fim, pôde-se perceber que a gestão dos processos juntamente com a análise dos custos baseado no método $A B C$, possibilita uma melhoria contínua nos processos organizacionais, pois, por meio desta análise, consegue-se verificar os desperdícios e realocar as tarefas de modo que se reduza tais problemas do processo.

\section{CONSIDERAÇÕES FINAIS}

Esta pesquisa teve por objetivo definir a modelagem na visão de Business Process Management (BPM) de um processo de trabalho em uma empresa prestadora de serviços contábeis e seu custeamento por meio do método $A B C$. Para que esse fosse alcançado, realizou-se a coleta de informações por meio de relatórios e entrevistas não estruturadas, as quais sustentaram o desenvolvimento da modelagem do processo de trabalho do arquivamento da Empresa Alfa, bem como a realização do custeamento de tal atividade.

Verificou-se ainda pela fundamentação teórica que apoia este trabalho e por meio do estudo de caso realizado, que a implementação da gestão de custos sobre a visão de processos de trabalho (BPM) realmente apresenta vantagens em relação à visão sistêmica da organização. Dentre essas vantagens identificadas podem ser citadas a facilidade em modelar e principalmente remodelar atividades complexas por meio de processos de trabalho, para se obter vantagens, tais como financeiras, aplicando o método de custeio por processo de trabalho. Essas vantagens observadas corroboram com a ideologia do custeio ABC desenvolvida por Coopers e Kaplan. 
Gestão de custos na visão de Business Process Management: uma simulação com o método de custeio Activity Based Costing Marino Luiz Eyerkaufer, Rodrigo Rengel, Rodrigo Wamser

Contudo, também foram identificadas algumas limitações da abordagem da modelagem e custeio de cada processo do estudo de caso realizado. Inicialmente, os pesquisadores identificaram um grau de subjetivismo durante as entrevistas com funcionários para mensuração do tempo de realização das atividades e para a identificação das variáveis que as afetam. Além disso, algumas atividades dentro do processo escolhido não podem ser padronizadas, o que impede sua modelagem por meio das equações de tempo. Algumas limitações observadas no desenvolvimento do modelo, contudo, não são derivadas da abordagem do método de custeio $A B C$ ou na gestão por processos (BPM), mas, sim, de peculiaridades do caso estudado.

Salienta-se que uma das principais vantagens apresentadas no estudo de caso, foi a realocação de atividades, por meio de análises dos processos, visando redução de custos internos e a facilidade de controle interno. Apresentou-se dessa maneira uma contribuição prática à empresa objeto de estudo. Tal pesquisa contribui ainda no âmbito teórico por proporcionar uma visão complementar entre o BPM e o $A B C$, possibilitando tal identificação das atividades e processos de trabalho desalinhados ou desnecessários.

Por fim, ressaltam-se algumas limitações da pesquisa descrita neste trabalho. Por se tratar de um estudo de caso, não é possível a generalização dos resultados obtidos em uma abordagem indutiva. Ademais, o fato de se tratar de um único processo na empresa compromete a comparabilidade com possíveis resultados encontrados nos demais departamentos. Estudos futuros poderiam desenvolver pesquisas englobando todos os processos internos de empresas do mesmo ramo de atuação, a fim de corroborar ou não os resultados apresentados neste trabalho. Estudos futuros poderiam ainda adotar uma visão comparativa decorrentes da aplicação dos métodos em organizações do mesmo segmento, permitindo, assim, evidenciar ainda mais as potencialidades ou deficiências da aplicabilidade do método. 
Gestão de custos na visão de Business Process Management: uma simulação com o método de custeio Activity Based Costing Marino Luiz Eyerkaufer, Rodrigo Rengel, Rodrigo Wamser

\section{REFERÊNCIAS}

Abbas, K., Gonçalves, M. N., \& Leoncine, M. (2012). Os métodos de custeio: vantagens, desvantagens e sua aplicabilidade nos diversos tipos de organizações apresentadas pela literatura. Contexto, 12(22), 145-159.

BPM CBOK. (2013). Business Process Management Common Body of Knowledge, 3. Primeira Liberação em Português. ABPMP.

Anand, M. (2004). A review of research on the theory \& practice of cost management. South Asian Journal of Management, 11(1), 59-95.

Bonacim, C. A. G., \& Araujo, A. M. P. D. (2010). Gestão de custos aplicada a hospitais universitários públicos: a experiência do Hospital das Clínicas da Faculdade de Medicina de Ribeirão Preto da USP. Revista de Administração Pública, 44(4), 903931.

Brasil. (2006). Lei complementar $n$ 123, de 14 de dezembro de 2006. Diário Oficial da República Federativa do Brasil, Poder Executivo, Brasília, DF, 15 de Dez de 2006, Seção 1, p. 1.

Bruni, A. L.; Famá, R. (2004). Gestão de custos e formação de preços. São Paulo: Atlas.

Burlton, R. T. (2001). Business Process Management: profiting from process. Indianapolis: Sams.

Carareto, E. S., Jayme, G., Tavares, M. P. Z., \& Vale, V. P. (2006). Gestão estratégica de custos: custos na tomada de decisão. Revista de Economia da UEG, 2(2), 1-24.

Ciotti, L. M. M. (2011). Gestão estratégica de custos e resultados na Comercial Tijolinhos. (Trabalho de conclusão de curso). Lato Sensu em Controladoria e Gestão Empresarial do Departamento de Ciências Administrativas, Contábeis, Econômicas e da Comunicação da Unijuí. - Universidade Regional do Noroeste do Estado do Rio Grande do Sul, UNIJUI, ljuí, RS.

Coelho, A, M, M, S. (2011). Os Sistemas de Custeio e a Competitividade da Empresa. (Dissertação de Mestrado). Contabilidade e Finanças. Instituto Superior de Contabilidade e Administração do Porto, Portugal.

Cogan, S. (2010). Custeio ABC e outras sistemáticas de custeio: uma proposta de aperfeiçoamento. Sociedade, Contabilidade e Gestão, 1(1). 
Gestão de custos na visão de Business Process Management: uma simulação com o método de custeio Activity Based Costing

Marino Luiz Eyerkaufer, Rodrigo Rengel, Rodrigo Wamser

Coronetti, J., Beuren, I. M., \& Sousa, M. A. B. (2012). Os métodos de custeio utilizados nas maiores indústrias de Santa Catarina. Gestão.org.,10, 02, 324-343.

Costa, G. S. C. S. (2013). Gerenciamento de Custos Econômicos Aplicado a Processos sob um Enfoque Orientado a Objetos. Registro Contábil, 4(2), 123-138.

Cunha, L. C., Borgert, A., \& Ferrari, M. J. (2015). Gestão estratégica de custos nos cursos de graduação em ciências contábeis das instituições de ensino superior do Estado de Santa Catarina. Revista Catarinense da Ciência Contábil, 14(41), 61-73.

De Sordi, J. O. (2005). Gestão de processos: uma abordagem da moderna administração. São Paulo: Saraiva.

Diehl, C. A., Capalonga, G., Souza, M. D., \& Zanini, F. (2009). As estratégias percebidas sob o enfoque teórico do posicionamento, da visão baseada em recursos, da missão e da tipologia: Um Estudo Com Empresas Gaúchas. In Congresso USP de Controladoria e Contabilidade. São Paulo.

Dos Santos, W. O., \& Robaina, D. T. (2014). O Impacto do Gerenciamento de Processos de Negócio (bpm) nos Custos Organizacionais. Artigo apresentado no Simpósio de Excelência em Gestão e Tecnologia (SEGeT).

Gasparetto, V. (1999). Uma discussão sobre a seleção de direcionadores de custos na implantação do custeio baseado em atividades. (Dissertação de Mestrado). PósGraduação em Engenharia de Produção. Universidade Federal de Santa Catarina, UFSC. Florianópolis, Santa Catarina, SC.

Lagioia, U. C. T., Ribeiro Filho, J. F., Falk, J. A., Libonati, J. J., \& Lopes, J. E. D. G. (2008). A gestão por processos gera melhoria de qualidade e redução de custos: o caso da unidade de ortopedia e traumatologia do hospital das clínicas da Universidade Federal de Pernambuco. Revista Contabilidade \& Finanças, 19(48), 77-90.

Luz Planilhas Empresariais. Contabilidade de custos, o que é e como fazer. Recuperado de: https://blog.luz.vc/o-que-e/contabilidade-de-custos/. Acesso em 26/jul/2017.

Machado, D. G., \& Souza, M. A. (2006). Análise das relações entre a gestão de custos e a gestão do preço de venda: um estudo das práticas adotadas por empresas industriais conserveiras estabelecidas no RS. Revista Universo Contábil, 2 (1), 4260.

Martins, E. (2003). Contabilidade de custos. (9a ed.). São Paulo: Atlas. 
Gestão de custos na visão de Business Process Management: uma simulação com o método de custeio Activity Based Costing

Marino Luiz Eyerkaufer, Rodrigo Rengel, Rodrigo Wamser

Megliorini, E. (2012). Custos: análise e gestão. (3a ed.). São Paulo: Pearson Prentice Hall.

Nakagawa, M. (2001). Custeio Baseado em Atividades. São Paulo: Atlas.

Oliveira, D. P. R. (2010). Planejamento estratégico: conceitos, metodologia e prática. (28a ed.). São Paulo: Atlas.

Perez Junior, H. J., Oliveira, L. M., \& Costa, R. G. (2003). Gestão Estratégica de Custos. São Paulo: Altas.

Pizza, W. R. (2012). A metodologia Business Process Management (BPM) e sua importância para as organizações. (Monografia). Tecnologia em processamento de dados. FATEC-SP, São Paulo, SP.

Porter, M. E. (1980). Competitive strategy: techniques for analysing industries and competitors. New York: Free Press.

Porter, M. E. (1989). Vantagem competitiva: criando e sustentando um desempenho superior. Rio de Janeiro: Campus.

Raupp, F. M., \& Beuren, I. M. (2006). Metodologia da Pesquisa Aplicável às Ciências. Como elaborar trabalhos monográficos em contabilidade: teoria e prática. São Paulo: Atlas, 76-97.

Raupp, F. M., \& Beuren, I. M. (2011). Metodologias de custos utilizadas nas maiores indústrias do setor têxtil do Estado de Santa Catarina. Revista Ciências Administrativas, 17(3), 973-1000.

Santos, J. L.; Schmidt, P.; Pinheiro, P. R.; Nunes, M. S. (2006). Fundamentos de Contabilidade de custos. São Paulo: Atlas S/A.

Scucuglia, R. Gestão por Processos (BPM): uma mudança de filosofia gerencial. Recuperado de: http://www.portaldecontabilidade.com.br/tematicas/contabilidadeetecnologia.htm. Acesso em 01/jul/2017

Silva, C. L. (1999). Gestão estratégica de custos na cadeia de valor: o custo meta na cadeia de valor. Revista FAE, 2 (2), 17-26.

Silva, Z. D., Ramalho, W., \& Jordão, R. V. D. (2015). Desenvolvimento de um Instrumento Gerencial de Custo para uma Gestão Estratégica em Empresas de 
Gestão de custos na visão de Business Process Management: uma simulação com o método de custeio Activity Based Costing Marino Luiz Eyerkaufer, Rodrigo Rengel, Rodrigo Wamser

Serviços Contábeis: Um Estudo Multicasos. Revista de Gestão e Projetos-GeP, $6(2), 42-55$.

Tabosa, C. De M, Júnior, A. F. S, Rodrigues, M. V, \& Pinheiro, C. D. P. (2008). Modelo de um sistema híbrido de Gestão de custos integrando Custeio direto e custeio Baseado em atividades: um Estudo de caso em uma empresa Do setor metalgráfico. Encontro Nacional de Engenharia de Produção, Rio de Janeiro, RJ, Brasil, 28.

Yin, R. K. (2005). Estudo de caso: planejamento e métodos. (3a ed.). Porto Alegre: Bookman.

Data de Submissão: 31/03/2020

Data de Aceite: 01/10/2020 